\title{
Does pre- and postoperative metronidazole treatment lower vaginal cuff infection rate after abdominal hysterectomy among women with bacterial vaginosis?
}

\author{
Per-Göran Larsson ${ }^{1}$ and Bodil Carlsson ${ }^{2}$ \\ ${ }^{1}$ Department of Obstetrics and Gynecology, Central Hospital, Skövde (Kärnsjukhuset), and \\ ${ }^{2}$ Department of Obstetrics and Gynecology, Linköpings Universitet and Division of Clinical Microbiology, \\ Linköpings Universitet, Linköping, Sweden
}

Objective: Bacterial vaginosis (BV) is a known risk factor for postoperative infection following abdominal hysterectomy. Vaginal bacterial flora scored as intermediate has been shown to have the same risk of postoperative infection as BV.

Methods: Women undergoing total abdominal hysterectomy for benign diseases were open-randomized according to Zelen to either treatment with metronidazole rectally for at least 4 days or no treatment. At the preoperative gynecological examination a vaginal smear was collected and Gram stained. Women with BV or intermediate flora were merged to one group called abnormal vaginal flora.

Results: In total 213 women were randomized to treatment or no treatment. After exclusion of 7 I women, 142 women were eligible for analysis. Among the 59 women diagnosed with abnormal vaginal flora there were no vaginal cuff infections in the treated arm, compared with $27 \%$ in the 'no treatment' arm $(p<0.01)$. Treatment also reduced the vaginal cuff infection rate from 9.5 to $2 \%$ among the 83 women with lactobacilli flora. However, this difference was not statistically significant. Treatment had no effect on the rate of wound infections. Intention-to-treat analysis showed a significant reduction in vaginal cuff infections among women randomized to treatment.

Conclusion: Pre- and postoperative treatment for at least 4 days with metronidazole rectally reduces significantly vaginal cuff infection among women with abnormal vaginal flora.

Key words: Bacterial Vaginosis; Abdominal Hysterectomy; Postoperative Infection;

TREATMENT; INTERMEDiATE FLORA

Total abdominal hysterectomy is the most common procedure for removing the uterus. Preoperative administration of antibiotics has been recommended for vaginal hysterectomy, but not always for abdominal hysterectomy ${ }^{1}$. Results from a meta-analysis ${ }^{2}$, published in 1993 , showed that $21.1 \%$ of patients who did not receive antibiotic prophylaxis had a serious infection after abdominal hysterectomy, whereas only $9 \%$ of women given preoperative antibiotics prior to abdominal hysterectomy had serious postoperative infection. This practice of preoperative antibiotic administration has not been accepted as good clinical practice in Scandinavia, due to the fact that several Scandinavian studies showed little effect of preoperative antibiotic prophylaxis on postoperative

Correspondence to: P-G. Larsson, MD, PhD, Department of Obstetrics and Gynecology, Kärnsjukhuset, S-541 85 Skövde, Sweden. Email: p-g.larsson@vgregion.se 
morbidity ${ }^{3-6}$. Mittendorf ${ }^{2}$ showed that metronidazole was the drug of choice for prophylactic antibiotics, but other authors have suggested second-generation cephalosporins as a better choice.

Bacterial vaginosis $(\mathrm{BV})$ is a condition in which the vaginal flora is characterized by a 1000 10 000-fold increase in concentration of various bacteria, including anaerobic bacteria, Gardnerella vaginalis, mycoplasmas and Mobiluncus, which primarily affect menstruating women. However, the vaginal flora of women without BV differs considerably, from a lactobacilli-dominated flora to having no identifiable lactobacilli. Women with no or few lactobacilli seen by microscopy, and not having a diagnosis of $\mathrm{BV}$, are said to have 'intermediate flora', i.e. a flora between normal and $\mathrm{BV}^{7}$. Diagnostic difficulties regarding vaginosis arise when trying to differentiate BV from intermediate flora and not so much when differentiating between normal lactobacilli flora and BV or intermediate flora. Intermediate flora seems to be of similar clinical significance as $\mathrm{BV}^{8-10}$. Thus, it has been suggested that intermediate flora and BV should be merged to one single group called 'abnormal vaginal flora'11.

Four studies are available concerning the risk of postoperative pelvic infection after abdominal hysterectomy among women with $\mathrm{BV}^{12-15}$. The first study ${ }^{12}$ comprised 161 women scheduled for abdominal hysterectomy for benign reasons. Women with BV had a relative risk (RR) of 3.4 (95\% confidence interval (CI) 1.5-6.7) for cuff cellulitis compared with women who had a negative preoperative diagnosis of $\mathrm{BV}$. In the second study ${ }^{13}, 70$ women undergoing abdominal hysterectomy for benign reasons with exclusion of postmenopausal women showed a RR of 4.4 (95\% CI 1.4-13.3) for developing postoperative infection if the women had BV. A nation-wide study from Sweden ${ }^{14}$ found a RR of 3 (95\% CI 1.3-7) for postoperative infection after abdominal hysterectomy for benign reasons among women with $\mathrm{BV}$ compared to women without $\mathrm{BV}$. In a recently published study Lin and co-workers ${ }^{15}$ found an increased risk of postoperative infection after all major gynecological surgery if the patients had a preoperative diagnosis of BV. In that study women diagnosed with an intermediate flora had no increased risk of postoperative infections.

The aims of this study are to investigate the effect of pre- and postoperative metronidazole treatment regarding the postoperative infection rate among women with abnormal vaginal flora compared to women with lactobacilli flora.

\section{SUBJECTS AND METHODS}

\section{Study population}

The current study included women undergoing an elective total abdominal hysterectomy for benign reasons from 1992 to 1996, less than 50 years of age or if over 50 still menstruating. We enrolled 213 patients into the study. Seventy-one of these were excluded (Table 1). Exclusion criteria were preoperative antibiotic use within one month prior to surgery, surgery for malignant disease and operations that for other reasons required preoperative antibiotics. Twenty-two patients were excluded for missing or inadequate vaginal smears, mostly

Table I Excluded patients $(n=7 \mathrm{I})$

\begin{tabular}{lr}
\hline Wrong randomization & $n$ \\
\hline Supra vaginal hysterectomy & 10 \\
Postmenopausal & 1 \\
Declined study entry & 15 \\
Preoperative antibiotics & 5 \\
Malignant surgery & 3 \\
Total & 34 \\
\hline Operative complications & $n$ \\
\hline Preoperative antibiotics & 6 \\
Re-operation & 1 \\
Other intestinal surgery that required antibiotics & 2 \\
Total & 9 \\
\hline Postoperative complications & $n$ \\
\hline Antibiotics for pneumonia, cholecystis, ileus & 4 \\
Allergic reaction to metronidazole & 2 \\
Total & 22 \\
\hline Smear problems & 6 \\
\hline Missing or uninterrupted smear, treatment & 13 \\
Missing or uninterrupted smear, no treatment & 9 \\
\hline
\end{tabular}


due to interfering menstrual blood. We limited the study to menstruating women, as the concept of $\mathrm{BV}$ and intermediate flora are poorly understood regarding postmenopausal women. No consideration was taken as to whether the women were menstruating due to sequential hormone replacement therapy (HRT) or endogenous hormone production.

\section{Patient evaluation}

Randomization was done according to Zelen ${ }^{16}$, using sealed envelopes in blocks of ten patients and carried out before informed consent. The nurse asked patients at preoperative registration if they wanted to join the study, before the patients met the operating doctor at the final examination. Thus, women randomized to the non-treatment group were not asked to join the study, as no antibiotic treatment is the normal procedure at the clinic. Women randomized to the treatment group were asked to join the study and receive treatment. In some cases the operation method was changed from the original planned total abdominal hysterectomy to other types of hysterectomies after randomization and the women were then excluded from the study. The normal preoperative procedure included a pelvic examination by the attending physician where vaginal fluid was obtained from the posterior fornix, placed on a microscope slide, air-dried and stored at room temperature. Demographic data, medical history and physical findings were recorded on standardized study forms. The vaginal slide was Gram stained and analyzed according to Nugents scoring system ${ }^{17}$ by one trained examiner without knowledge of the postoperative outcome and all slides were blindly batch analyzed after the study was finished. No evaluation of the vaginal flora was done preoperatively and the doctor had no knowledge of the vaginal status of the patient during operation or at postoperative care. If postoperative infection occurred this was recorded by the attending physician on the special form for the study.

\section{Definitions}

BV was defined according to Nugent's criteria ${ }^{17}$ as absence of lactobacilli and presence of a Gardnerella morphotype flora, with a Nugent's score of $7-10$. Women with a score of $0-3$ were regarded as normal and women with a score of 4-6 as intermediate. Women with a diagnosis of BV or intermediate flora were merged to a group called abnormal vaginal flora.

Women were defined as having a postoperative vaginal cuff infection if they had three or all of the following four signs during gynecological examination: (1) increased abdominal pain after the second day of operation; (2) temperature more than $38^{\circ} \mathrm{C}$ rectally after the third day; (3) malodorous discharge; and (4) induration or pain in the vaginal vault.

Wound infection was defined as induration and increased redness around the wound where antibiotic treatment or debridement where needed. If a woman had both vaginal cuff and wound infection she was recorded as a vaginal cuff infection. Urinary tract infection (UTI) was positive urinary culture with more than 100000 bacteria/ml.

Unexplained fever was defined as temperature above $38^{\circ} \mathrm{C}$ after the third day of operation without any other known diagnosis. All medical records were scrutinized before the vaginal smears were investigated.

\section{Treatment and follow-up}

On the evening before the operation the women were given $1 \mathrm{~g}$ metronidazole rectally (Flagyl ${ }^{\mathrm{TM}}$ suppositories $1 \mathrm{~g}$, Rhône-Poulenc, Rorer, Helsingborg) and the treatment was continued every evening until the patient was discharged from the gynecological ward or until any sideeffects were noticed. The most common treatment for BV is 7-day treatment with oral metronidazole with a dose of $500 \mathrm{mg}$ BID. Because nausea is a well known side-effect of orally administrated metronidazole, we chose rectal administration which has a slower reabsorption rate than oral administration, but the dose of $1 \mathrm{~g}$ will give the same serum concentration as oral treatment. The gynecologist on duty in the ward performed the operation. All the surgeons were specialists in gynecology or were supervised by a senior doctor. Total abdominal hysterectomy was performed either by a low Cohen incision or low longitudinal incision and the vaginal cuff was closed. All other 
surgical procedures done at the same time were recorded, i.e. bilateral salpingooophorectomy (BSO) or incontinence surgery such as Burch procedure.

Women over 50 years of age received antithrombotic prophylaxis with low molecular heparin. At the operating room the operating nurse routinely cleaned the vagina preoperatively with chlorhexidine solution just before the start of the operation. A catheter (KAD) was left in the bladder until the second day postoperatively. Patients were free to eat as soon as they felt able. All women were offered a follow-up visit 4-6 weeks after the operation in the gynecological outpatient clinic. All medical records from the follow-up visits were scrutinized before the results from the vaginal examinations were added.

\section{Statistics}

The Fisher exact test was used when less then 100 observations, otherwise the chi square $\left(\chi^{2}\right)$ test was used, together with RR with $95 \%$ CI. A logistic regression analysis and variance analysis was performed.

\section{Ethics}

The study was approved by the regional ethics committee at the University of Gothenburg and the 'Svenska läkemedelsverket' (Swedish Medical Products Agency). Women gave verbal informed consent to participate in the study.

\section{RESULTS}

The study population included 142 patients. There were no differences in the demographic factors between the treated and not treated group (Table 2). There were no differences in operating time, preoperative bleeding or preoperative blood hemoglobin, and a logistic regression was performed using these variables which showed that preoperative hemoglobin, weight, operating time and bleeding had no influence on the postoperative infection rate for all three groups of women (all women, non-treatment group and treatment group). The indication for the hysterectomy was dysfunctional uterine bleeding (DUB) or fibroids in more than $80 \%$ of cases.

The treatment consisted of metronidazole rectally from the night before operation until discharge from the hospital or at minimum 4 days of treatment. Side-effects such as nausea were noticed among eight $(12 \%)$ of the 67 treated women. These eight women discontinued the treatment in advance but still after the required 4 days of treatment. The mean treatment time was 5.8 days with a range of 4-13 days. Two patients had an allergic reaction after the first dose of treatment and were excluded from the study (Table 1).

The microscopy of the vaginal flora showed that $41.5 \%$ of the women had abnormal vaginal flora (17.6\% of women with BV and $23.9 \%$ with intermediate flora) and $58.5 \%$ had normal lactobacilli flora.

The mean time for the postoperative follow-up visit for non-infected women was 6.4 weeks with a range of 4-12 weeks, except for two patients who did not come to the scheduled visit. These two patients were interviewed by telephone. Fourteen women had a vaginal cuff infection, 13 of whom were diagnosed before discharge from the hospital. Only five of the ten wound infections had been detected before leaving the hospital. Of 25 UTI, 21 were known before the patients left the

Table 2 Background factors

\begin{tabular}{lcccccc}
\hline & \multicolumn{2}{c}{$\begin{array}{c}\text { All women } \\
(n=142)\end{array}$} & \multicolumn{2}{c}{$\begin{array}{c}\text { Treatment group } \\
(n=67)\end{array}$} & \multicolumn{2}{c}{$\begin{array}{c}\text { Non-treatment group } \\
(n=75)\end{array}$} \\
\cline { 2 - 7 } & Mean & Range & Mean & Range & Mean & Range \\
\hline Age (years) & 46 & $33-58$ & 46 & $33-58$ & 45 & $33-57$ \\
Weight $(\mathrm{kg})$ & 71 & $44-110$ & 73 & $44-110$ & 70 & $51-93$ \\
Operating time (minutes) & 100 & $43-210$ & 99 & $43-210$ & 102 & $50-190$ \\
Preoperative bleeding $(\mathrm{ml})$ & 578 & $75-2400$ & 580 & $100-2400$ & 576 & $75-2300$ \\
Preoperative hemoglobin $(\mathrm{g} / \mathrm{l})$ & 127 & $83-154$ & 127 & $83-146$ & 127 & $89-154$ \\
\hline
\end{tabular}


hospital. Fifteen patients left the hospital with a slight increase in body temperature, however without a specific diagnosis i.e. unexplained fever and with no treatment. Four (26\%) of these patients came back with a UTI but none of these patients had an undetected wound or cuff infection.

There was no significant difference in wound infection rate among the treated or non-treated women (Table 3). The frequency of cuff infection decreased from 27 to $0 \%$ in women with an abnormal vaginal flora that were treated with metronidazole $(p<0.05)$. This finding was even more pronounced among women with $\mathrm{BV}$ as the vaginal cuff infection decreased from 35.7 to $0 \%$ in the metronidazole group $(p<0.05)$. The vaginal cuff infection rate only decreased from 9.5 to $2 \%$ for women with normal lactobacilli flora (not significant) (Table 3). There were no differences in UTI rate between the various vaginal flora groups, and treatment with metronidazole did not reduce the incidence of postoperative UTI.

This study was performed when long postoperative hospital care after abdominal hysterectomies was common clinical practice. The mean time for hospital care was 5.8 days (standard deviation, SD 1.7) for women with no infection and 6.8 days (SD 3.5) for women with wound infection. Women with UTI stayed in hospital for 6.7 days (SD 1.7). Women with cuff infections had a mean hospital stay of 8.8 days (SD 2.4). Variance analysis with Tukey's pairwise comparisons showed statistically significant longer hospital care only for vaginal cuff infection $(p<0.01)$.

Intention-to-treat analysis for all 142 women showed a significant difference regarding cuff infections: one cuff infection (1.5\%) in the treated group versus 14 cuff infections (17\%) in the non-treated group ( $p<0.05, \chi^{2}$ test). The relative risk of postoperative cuff infection was significantly lower in the treated group versus the non-treated group (RR 0.1, 95\% CI 0.01-0.6). No difference was seen regarding the wound infections or UTI.

\section{DISCUSSION}

Bacterial vaginosis $(\mathrm{BV})$ is a condition in which the vaginal flora is characterized by a 1000-10 000fold increase in concentrations of various

Table 3 Infection rate among women after abdominal hysterectomy and treatment with pre- and postoperative metronidazole I g rectally for a minimum of 4 days

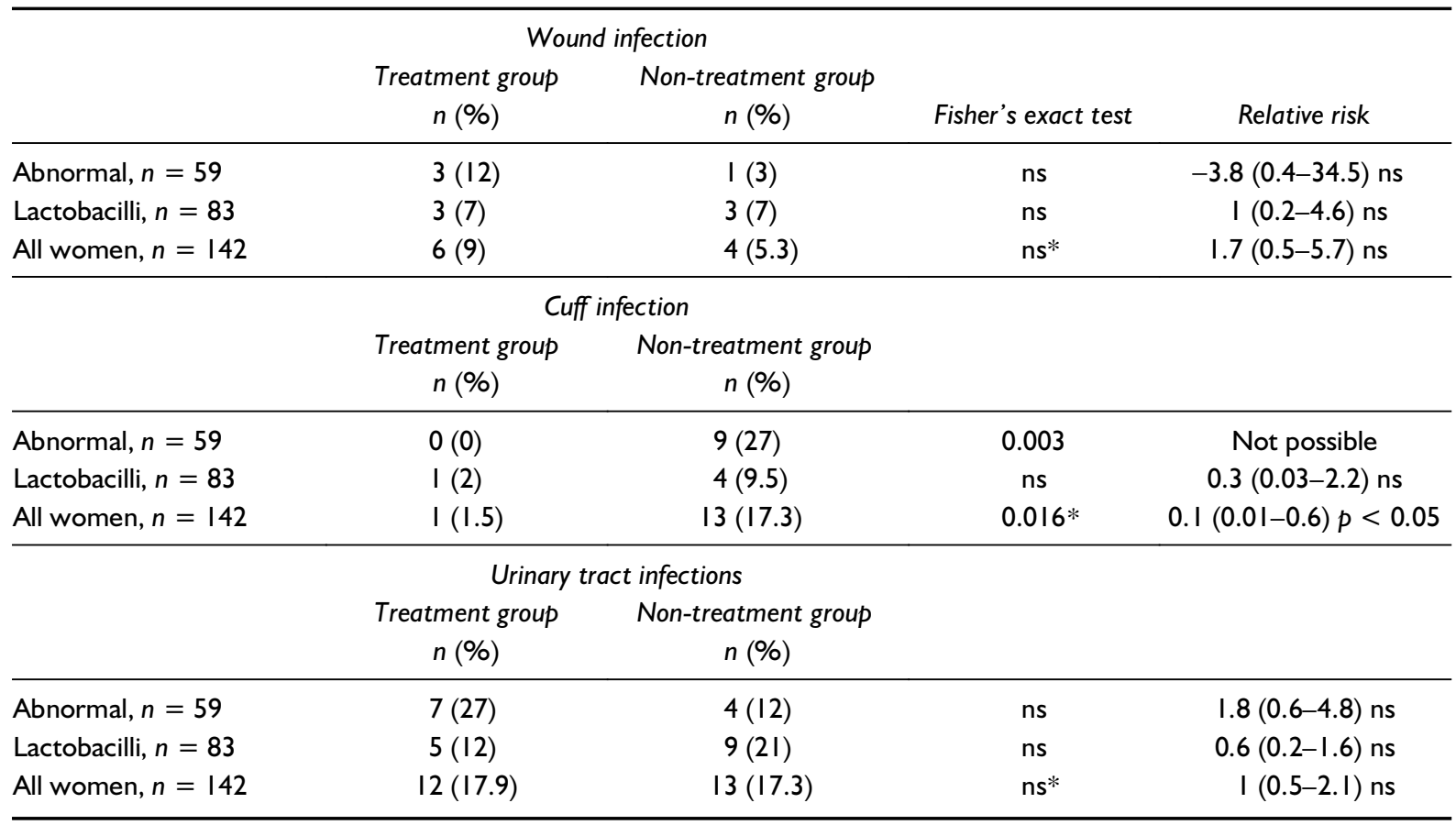

*Fisher's exact test except for 'all women': $\chi^{2}$ test was used as there were more than 100 observations; ns, not significant 
anaerobic bacteria. However, the vaginal flora of women with 'intermediate flora' has an increased concentration of bacteria of up to 1000 times $^{7}$. Houang and co-workers ${ }^{18}$ showed that the magnitude of bacteria contamination during operation is an important determinant in the development of postoperative infection after abdominal hysterectomy. This might be the theoretical explanation of the increased risk of postoperative infection in women with either BV or intermediate flora ${ }^{8-10}$ as it might be the increased concentration of bacteria rather than the pathogenic potential of the bacteria that causes the postoperative infection. When diagnosing $\mathrm{BV}$ it is often most difficult to make a clear distinction between full-blown BV and the intermediate flora. This is apparent when using Nugent scoring. According to Nugent's scoring system a score of six is given if there are one-four lactobacilli per high power field even if there are hundreds or thousands of small bacteria in the same high power field. A slide with that many small bacteria will also often have clue cells but this is something that Nugent's scoring system does not take into account. This is the reason why intermediate flora and BV in this study are merged into one group called abnormal vaginal flora. This merging was successful in an earlier study where women with lactobacilli flora did not benefit from preoperative clindamycin treatment before legal abortion, in contrast to women with abnormal vaginal flora defined as $\mathrm{BV}$ or intermediate flora ${ }^{10}$. To simplify the diagnosis in a practical clinical aspect it might be helpful to focus on the presence of lactobacilli. Women with a microscopic finding of vaginal flora of normal lactobacilli will receive no treatment whereas women in absence of normal lactobacilli flora will receive preoperative treatment.

Lin and co-workers ${ }^{15}$ did not find any effect of preoperative antibiotic treatment in the intermediate group. This could be due to the fact that they did not exclude postmenopausal women. Postmenopausal women lack lactobacilli in the vaginal flora and will score as intermediate flora according to Nugent. An earlier nationwide study showed that postmenopausal women have a lower risk of postoperative infection than younger women $^{14}$. In this study we have therefore deliberately restricted menstruating or estrogen-replaced women as the concept of $\mathrm{BV}$ and intermediate flora are poorly understood in postmenopausal women.

Today, hospital care has shortened in most places and patients are discharged much earlier from hospital. In addition, a higher degree of hysterectomies today are done either laparoscopically or vaginally, both with considerably shorter hospital care than abdominal hysterectomies. This is important to bear in mind when postoperative infection rates are reported and compared, as many postoperative infections do not arise until after discharge from hospital and therefore will alter documented infection rates.

In an open randomized trial there are always some methodological weaknesses. When the doctor in charge of the patient knows whether or not the patient is treated it might influence the results: if a patient with a suspected mild infection received metronidazole preoperatively, the doctor may wait one day before diagnosis. And patients known to have not been given metronidazole preoperatively might be given other antibiotics sooner. It could therefore be easier to diagnose a postoperative infection if the women had no treatment. However this would have affected the infection rates in the treated and not-treated groups, and not the difference between lactobacilli or abnormal vaginal flora group as the diagnosis of the vaginal flora was unknown to the doctor. The definition of postoperative infection is subjective, as always in open clinical studies, and our frequency of postoperative infection could only be compared with other hospitals with caution. The subjective definition of postoperative infection would only reduce any difference in the treatment groups and would therefore weaken our results but not affect the conclusion of the study.

There are many different treatment regimens for BV. One commonly seen is $4 \mathrm{~g}$ metronidazole given as a single $2 \mathrm{~g}$ dose on day one and three. Because of this we required at least 4 days of treatment for evaluation of efficacy, i.e. $4 \mathrm{~g}$ metronidazole. Treatment with oral metronidazole is known to cause nausea, a well-known effect even of general anesthesia. Therefore we decided to administer metronidazole rectally instead of orally, to minimize exclusions due to medical events. 
Our study is the first intervention study of BV and intermediate flora among women operated with abdominal hysterectomy. We showed that women with BV or intermediate flora benefit from antibiotic treatment and should be offered treatment starting the day before operation. Although our intention-to-treat analysis showed that all women, even those with normal lactobacilli flora, would benefit from treatment to some degree, it is questionable whether women with normal lactobacilli flora should be given antibiotic treatment for four days. We found no effect on the frequency of wound infections or UTI. These results were expected as UTI and wound infections are mostly caused by aerobic bacteria resistant to metronidazole.

\section{ACKNOWLEDGMENTS}

This study was supported by the Rhône-Poulenc Rorer and grants from the 'FoU - Skaraborg' and 'FoU - VästraGötalands regionen'. We thank all doctors and nurses from the gynecological ward at the Central Hospital of Skövde (Kärnsjukhuset) for their support during the study.

\section{REFERENCES}

1. Hemsell DL, Heard MC, Nobles BJ, et al. Single-dose prophylaxis for vaginal and abdominal hysterectomy. Am J Obstet Gynecol 1987;157: 498-501

2. Mittendorf R, Aronson MP, Berry RE, et al. Avoiding serious infections associated with abdominal hysterectomy: a meta-analysis of antibiotic prophylaxis. Am J Obstet Gynecol 1993;169: 1119-24

3. Evaldson GR, Lindgren S, Malmborg AS, Nord CE. Single-dose intravenous tinidazole prophylaxis in abdominal hysterectomy. Acta Obstet Gynecol Scand 1986;65:361-5

4. Munck AM, Jensen HK. Preoperative clindamycin treatment and vaginal drainage in hysterectomy. Acta Obstet Gynecol Scand 1989;68:241-5

5. Mamsen A, Hansen V, Moller BR. A prospective randomized double-blind trial of ceftriaxone versus no treatment for abdominal hysterectomy. Eur $J$ Obstet Gynecol Reprod Biol 1992;47:235-8

6. Henriksson L, Colling-Saltin AS, Frick G, et al. Metronidazole prophylaxis to prevent infections after total abdominal hysterectomy. Acta Obstet Gynecol Scand 1998;77:116-9

7. Rosenstein IJ, Morgan DJ, Sheehan M, et al. Bacterial vaginosis in pregnancy: distribution of bacterial species in different Gram-stain categories of the vaginal flora. J Med Microbiol 1996;45:120-6

8. Morgan DJ, Wong SJ, Trueman G, et al. Can bacterial vaginosis influence fertility? Its increased prevalence in a subfertile population. Int J STD AIDS 1997;8(Suppl 1):19-20
9. Llahi-Camp JM, Rai R, Ison C, et al. Association of bacterial vaginosis with a history of second trimester miscarriage. Hum Reprod 1996;11:1575-8

10. Larsson PG, Platz-Christensen JJ, Dalaker K, et al. Treatment with $2 \%$ clindamycin vaginal cream prior to first trimester surgical abortion to reduce signs of postoperative infection: a prospective, double-blinded, placebo-controlled, multicenter study. Acta Obstet Gynecol Scand 2000;79:390-6

11. Ison CA. Bacterial vaginosis. Microbiology and epidemiology, introduction. Int J STD AIDS 1997; 8(Suppl 1):2-3.

12. Soper DE, Bump RC, Hurt WG. Bacterial vaginosis and trichomoniasis vaginitis are risk factors for cuff cellulitis after abdominal hysterectomy. Am J Obstet Gynecol 1990;163:1016-23

13. Larsson PG, Platz-Christensen JJ, Forsum U, Påhlson C. Clue cells in predicting infections after abdominal hysterectomy. Obstet Gynecol 1991;77: 450-2

14. Persson E, Bergstrom M, Larsson PG, et al. Infections after hysterectomy. A prospective nation-wide Swedish study. The Study Group on Infectious Diseases in Obstetrics and Gynecology within the Swedish Society of Obstetrics and Gynecology. Acta Obstet Gynecol Scand 1996;75: 757-61

15. Lin L, Song J, Kimber N, et al. The role of bacterial vaginosis in infection after major gynecologic surgery. Infect Dis Obstet Gynecol 1999;7:169-74

16. Zelen M. A new design for randomized clinical trials. N Engl J Med 1979;300:1242-5 
17. Nugent RP, Krohn MA, Hillier SH. Reliability of diagnosing bacterial vaginosis is improved by a standardized method of Gram stain interpretation. J Clin Microbiol 1991;29:297-301

RECEIVED 10/16/01; ACCEPTED 02/22/02
18. Houang ET, Ahmet Z. Intraoperative wound contamination during abdominal hysterectomy. J Hosp Infect 1991;19:181-9 


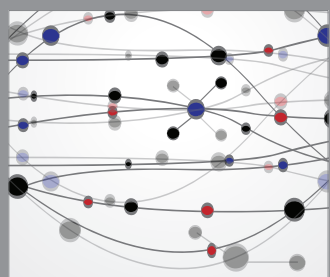

The Scientific World Journal
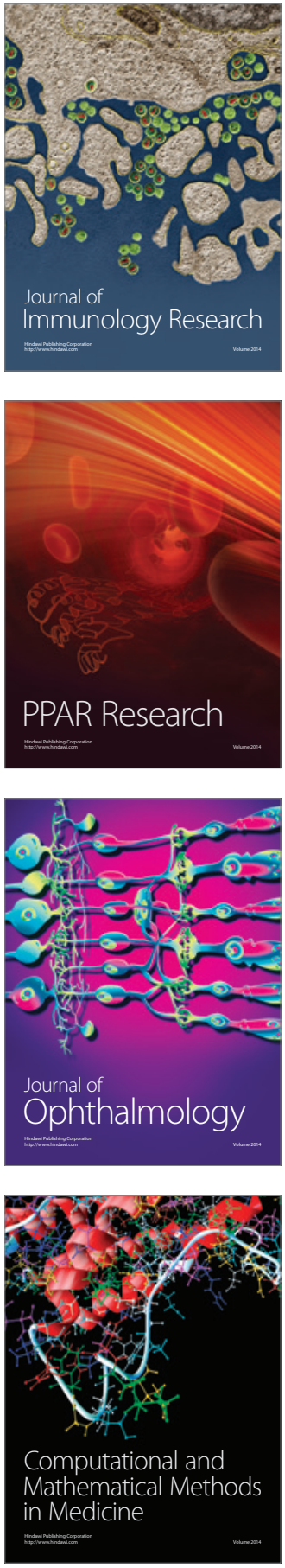

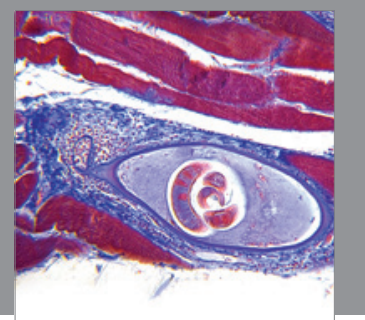

Gastroenterology

Research and Practice
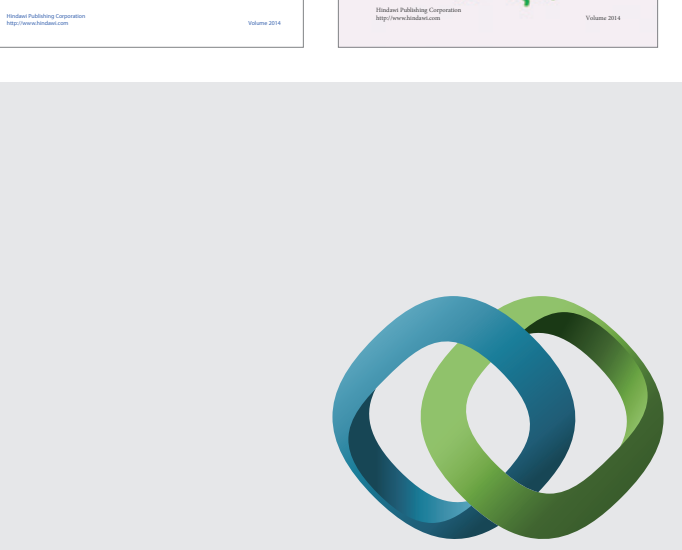

\section{Hindawi}

Submit your manuscripts at

http://www.hindawi.com
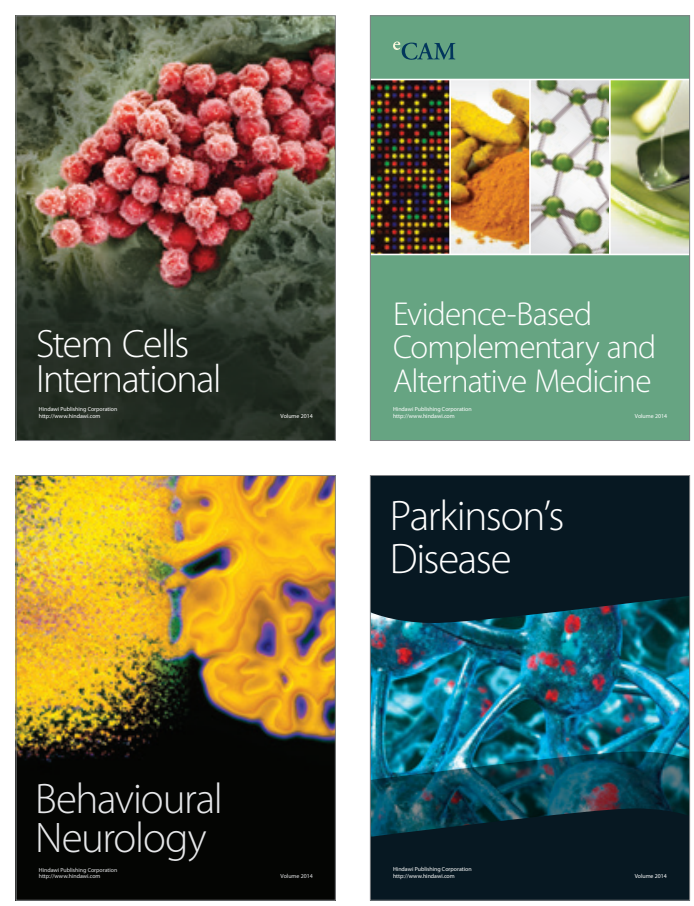

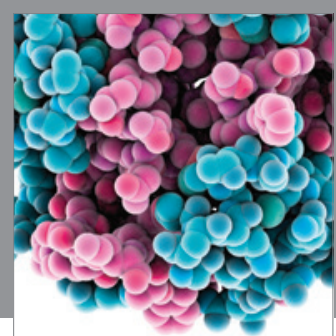

Journal of
Diabetes Research

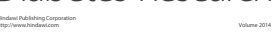

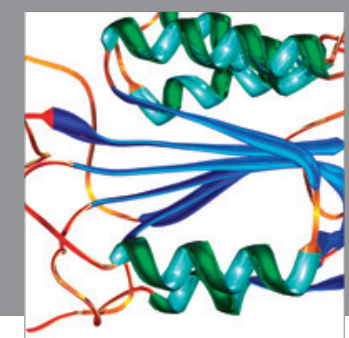

Disease Markers
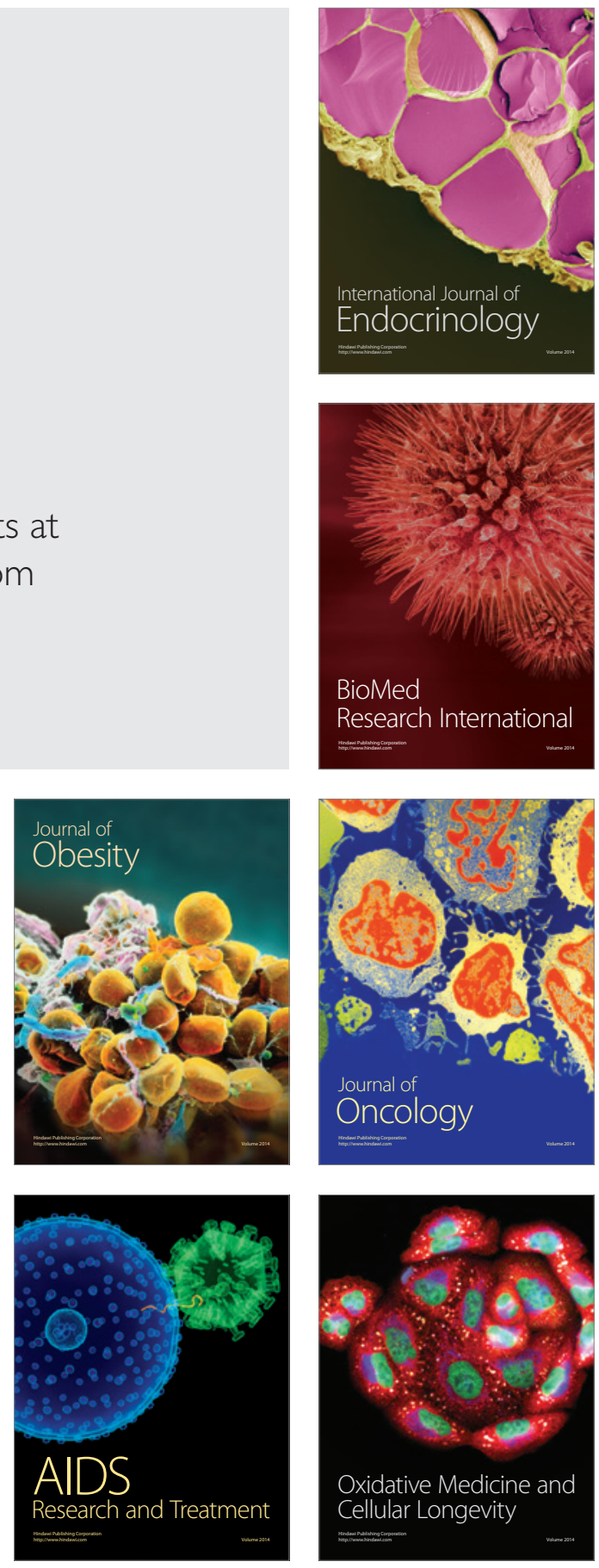